\title{
Bei RLS auf Nieren und Eisenspiegel achten
}

\author{
Eine systematische Literaturrecherche ergab eine hohe Assoziation von Eisenmangel und Nieren- \\ erkrankungen mit dem Restless-Legs-Syndrom (RLS). Dies hat auch therapeutische Konsequenzen.
}

_ In der Literatur sind eine Vielzahl von Assoziationen des RLS mit anderen Erkrankungen beschrieben, was die diagnostische Zuordnung zu einem primären oder einem möglich erscheinenden sekundären RLS erschwert.

Für ein systematisches Review wurden nun Studien zum Zusammenhang mit Eisenmangel, Anämie, Erkrankungen der Nieren, arterieller Hypertonie und kardiovaskulären Erkrankungen identifiziert. Bei den neurologischen Erkrankungen wurden Assoziationen mit Kopfschmerz, Schlaganfall, neurodegenerativen Erkrankungen, Polyneuropathie und multipler Sklerose untersucht. Die Wahrscheinlichkeit einer Assozia- tion mit dem RLS wurde jeweils als hoch, mittelstark und gering eingestuft.

Die Auswertung ergab lediglich für Eisenmangel und Nierenerkrankungen eine gesicherte Assoziation mit dem RLS. Bei den anderen Erkrankungen war der Zusammenhang gering, konnte aber letztendlich auch nicht ausgeschlossen werden. Unwahrscheinlich ist eine Assoziation mit einer Anämie ohne Eisenmangel sowie mit Lungenerkrankungen, multipler Sklerose, Kopfschmerzen, Schlaganfall, Narkolepsie und Ataxien.

- Trenkwalder C, Allen R, Högl B et al. Restless legs syndrome associated with major diseases: a systematic review and new concept. Neurology. 2016;86:1336-43

\section{KOMMENTAR}

Diese systematische Literaturrecherche ist sehr hilfreich, da basierend auf den Ergebnissen ein neues Modell für das $R L S$ vorgeschlagen wird. Demnach gibt es genetische Formen ohne Komorbidität, einen Überlappungsbereich, in dem das Syndrom durch andere Erkrankungen verstärkt wird, und wahrscheinlich einen geringen Anteil an Patienten, bei dem nur die Begleiterkrankung das RLS erklärt. Die praxisrelevante Erkenntnis ist, dass ein Eisenmangel beim RLS ausgeglichen und auch eine Nierenerkrankung soweit wie möglich behandelt werden sollten.

Prof. Dr. med. H.-C. Diener

\section{Bei diesem Mädchen häuft sich Knochenmatrix an}
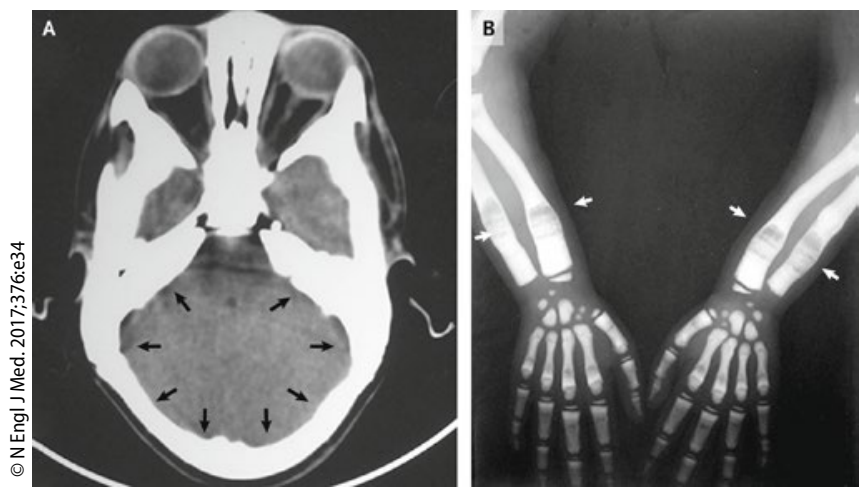

A: Verdickte Schädelknochen (Pfeile). B: Sklerosierte und aufgehellte Abschnitte der Ellen und Speichen (Pfeile).

Ein sechsjähriges Mädchen, dessen Eltern nah verwandt waren und das im im Alter von zwei Jahren Sehvermögen und Gehör verloren hatte, wurde zum Zahnarzt gebracht, weil es bislang erst einen einzigen Zahn hatte. Bei der Untersuchung fielen ein Strabismus, eine vorgewölbte Stirn, ein Hypertelorismus und ein Kleinwuchs auf. Die Serumspiegel von Kalzium und Phosphor waren ebenso wie die alkalische Phosphatase normal. Im Schädel-CT erkannte man diffus verdickte Schädelknochen (Abb. A). Im Röntgenbild beider
Unterarme zeigten sich die distalen Abschnitte von Elle und Speiche bandförmig sklerosiert und aufgehellt (Abb. B).

Die Befunde waren vereinbar mit der Diagnose einer Osteopetrose oder Marmorknochenkrankheit. Diese seltene Erkrankung geht mit erhöhter Knochendicke durch pathologische Anhäufung von Knochenmatrix einher. Genmutationen führen zu einer gestörten Osteoklastenfunktion und einem Versagen der Knochenresorption. Die genaue Ursache ist noch ungeklärt, der Erbgang kann autosomal-rezessiv oder autosomal-dominant sein. Zu den Komplikationen gehören - wie im vorliegenden Fall - Kompressionssyndrome der Hirnnerven an den Austrittsstellen aus dem Schädel, brüchige Knochen und Störungen der Blutbildung. Aufgrund der schwindenden Markräume kommt es zur extramedullären Blutbildung. Der ständige Aufbau von Knochenmatrix kann auch eine Hypokalzämie zur Folge haben.

Man begann eine Therapie mit Calcitriol. Geplant war weiterhin eine Behandlung mit Interferon gamma-1b, doch wurde das Mädchen von den Eltern nicht mehr vorgestellt. Die einzig wirksame therapeutische Option ist eine Knochenmarkstransplantation, da sich aus hämatopoietischen Stammzellen wieder normale Osteoklasten differenzieren können.

Prof. Dr. med. H. S. FüeßI

- Yaga U, Panta P. Osteopetrosis. N Engl J Med. 2017;376:e34 